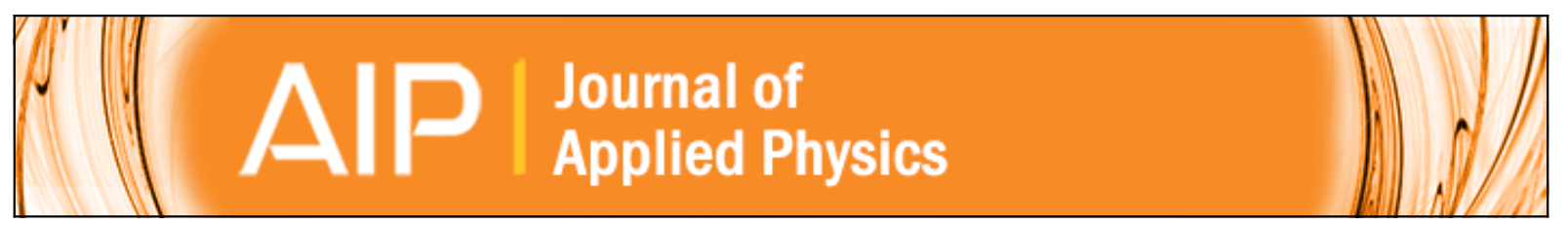

\title{
Magnetic properties and magnetocaloric effects in GdCo9Si2 compound with multiple magnetic phase transitions
}

Z. G. Zheng, X. C. Zhong, J. L. Zhang, Z. W. Liu, V. Franco, and D. C. Zeng

Citation: Journal of Applied Physics 113, 17 A938 (2013); doi: 10.1063/1.4799973

View online: http://dx.doi.org/10.1063/1.4799973

View Table of Contents: http://scitation.aip.org/content/aip/journal/jap/113/17?ver=pdfcov

Published by the AIP Publishing

\section{Articles you may be interested in}

Magnetic properties and magnetocaloric effect in the RCu2Si2 and RCu2Ge2 ( $\mathrm{R}=\mathrm{Ho}, \mathrm{Er})$ compounds

J. Appl. Phys. 115, 073905 (2014); 10.1063/1.4864419

Magnetic properties and magnetocaloric effects in Er3-xGdxCo intermetallic compounds

J. Appl. Phys. 113, 033908 (2013); 10.1063/1.4776742

Magnetic phase transitions and magnetocaloric properties of (Gd12-xTbx)Co7 alloys

J. Appl. Phys. 109, 07 A919 (2011); 10.1063/1.3551736

Magnetic and magnetocaloric properties of Gd6X2Si3 (X=Ni, Co) and Ln6Co2Si3 ( $\mathrm{Ln}=\mathrm{Pr}, \mathrm{La})$

J. Appl. Phys. 109, 07 A913 (2011); 10.1063/1.3544509

Thermopower behavior in the $\mathrm{Gd} 5$ ( $\mathrm{Si} 0.1 \mathrm{Ge} 0.9$ ) 4 magnetocaloric compound from 4 to $300 \mathrm{~K}$

J. Appl. Phys. 91, 4457 (2002); 10.1063/1.1459612

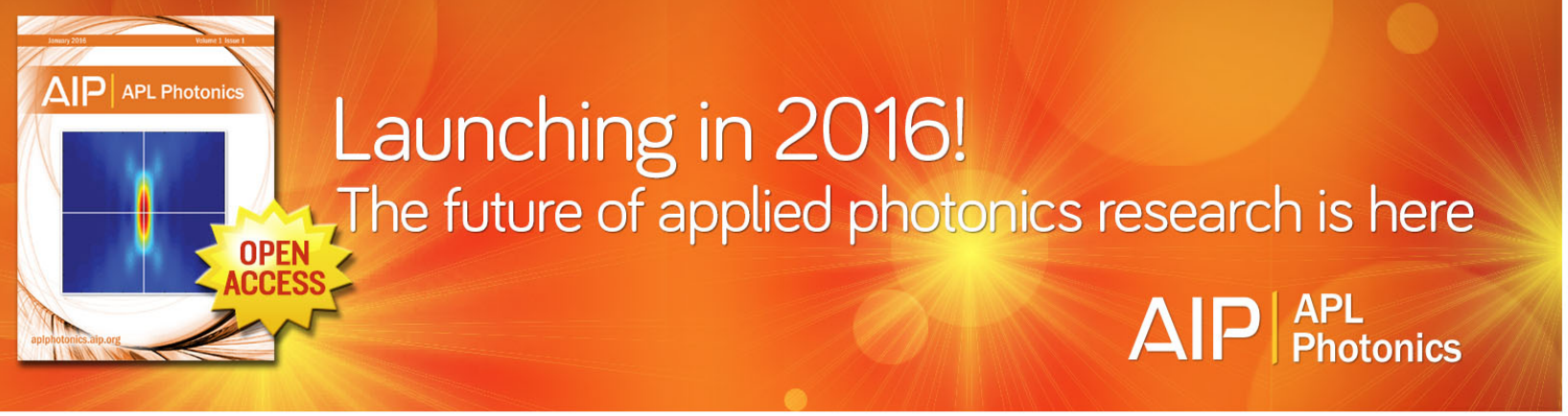




\title{
Magnetic properties and magnetocaloric effects in $\mathrm{GdCo}_{9} \mathrm{Si}_{2}$ compound with multiple magnetic phase transitions
}

\author{
Z. G. Zheng, ${ }^{1}$ X. C. Zhong, ${ }^{1}$ J. L. Zhang, ${ }^{2}$ Z. W. Liu, ${ }^{1, a)}$ V. Franco, ${ }^{3}$ and D. C. Zeng ${ }^{1, \text { a) }}$ \\ ${ }^{1}$ School of Materials Science \& Engineering, South China University of Technology, Guangzhou 510640 , \\ People's Republic of China \\ ${ }^{2}$ Department of Physics and Materials Science, City University of Hong Kong, Kowloon Tong, Hong Kong \\ ${ }^{3}$ Department of Condensed Matter Physics, ICMSE-CSIC, Sevilla University, P. O. Box 1065, 41080 Sevilla, \\ Spain
}

(Presented 18 January 2013; received 5 November 2012; accepted 22 January 2013; published online 10 April 2013)

\begin{abstract}
The structure and magnetic properties of polycrystalline $\mathrm{GdCo}_{9} \mathrm{Si}_{2}$ compound have been investigated. It has a $\mathrm{BaCd}_{11}$ structure and undergoes two magnetic phase transitions: an antiferromagnetic to ferrimagnetic transition occurring at $\sim 93 \mathrm{~K}$, and a ferrimagnetic to paramagnetic transition at $420 \mathrm{~K}$, which results in a positive and a negative magnetic entropy change, respectively. The two peak values of magnetic entropy change are -0.6 and 1.1 $\mathrm{J} \cdot \mathrm{kg}^{-1} \cdot \mathrm{K}^{-1}$ for $\Delta H=5 \mathrm{~T}$. Furthermore, there exists a metal-semiconductor transition temperature $\left(T_{\mathrm{P}}\right)$, below which the resistance increases with increasing temperature, while the semiconductor characteristic is observed above $T_{\mathrm{P}}$. The magnetic domain structures are characterized by stripe and grid structures $1 \mu \mathrm{m}$ wide. Although the MCE is small for applications, its study is useful to clearly understand the nature of multiple magnetic phase transitions in the $\mathrm{GdCo}_{9} \mathrm{Si}_{2}$ compound. (C) 2013 American Institute of Physics. [http://dx.doi.org/10.1063/1.4799973]
\end{abstract}

Magnetic materials with large magnetocaloric effect (MCE) have attracted considerable attention for potential applications in magnetic refrigeration. In recent years, excellent magnetocaloric properties have been observed in $\mathrm{Gd}$ and its compounds. ${ }^{1}$ Due to their large magnetic moments and low anisotropy, Gd-based compounds are definitely prime candidates for achieving large MCE. ${ }^{2}$ In a previous work, we have investigated the magnetic properties and magnetocaloric effect of compounds $\left(\mathrm{Gd}_{12-\mathrm{x}} \mathrm{Tb}_{\mathrm{x}}\right) \mathrm{Co}_{7}(\mathrm{x}=0,4$, and $8),{ }^{3}$ amorphous $\mathrm{Gd}_{4} \mathrm{Co}_{3}$ alloys, ${ }^{4}$ and $\mathrm{Gd}_{65} \mathrm{Mn}_{35-\mathrm{x}} \mathrm{Ge}_{\mathrm{x}}(\mathrm{x}=0$, 5 , and 10). ${ }^{5}$ All families exhibited large magnetic entropy change $\left(\Delta S_{\mathrm{M}}\right)$. However, the high contents of transition metals generally lead to the decrease of saturations because of their ferrimagnetic nature. This point is clearly seen from the high saturated moment of Co-deficient compounds, such as $\mathrm{Gd}_{5} \mathrm{CoSi}_{2}{ }^{6}$ The compound shows a ferromagnetic ordering at $168 \mathrm{~K}$ and has large MCE with adiabatic temperature change $\Delta T_{a d}=5.9 \mathrm{~K}$ and magnetic entropy change $-\Delta S_{M}$ $=8.7 \mathrm{~J} \cdot \mathrm{kg}^{-1} \mathrm{~K}^{-1}$ for a magnetic field change of $4.6 \mathrm{~T}$. Compared with Gd, transition metals show many practical advantages, such as lower material cost and larger corrosion resistance. There are previously published results for compounds in this system, such as $\mathrm{Gd}_{6} \mathrm{Co}_{2} \mathrm{Si}_{3}{ }^{7} \mathrm{GdCo}_{13-\mathrm{x}} \mathrm{Si}_{\mathrm{x}}{ }^{8}$ and $\mathrm{Gd}_{6} \mathrm{Co}_{1.67} \mathrm{Si}_{3}{ }^{9}$. They have gained more attention as potential highly efficient magnetocaloric materials and giant isotropic magnetostriction materials. ${ }^{10}$ This inspires us to investigate the magnetic behaviors of Co-rich compounds in the Gd-Co-Si system. However, except for above mentioned Gd-based compounds, only a few reports are available on the structure and magnetocaloric studies on this series. In this

\footnotetext{
a) Authors to whom correspondence should be addressed. Electronic addresses: zwliu@scut.edu.cn and medczeng@scut.edu.cn.
}

work, we have synthesized the ternary silicide $\mathrm{GdCo}_{9} \mathrm{Si}_{2}$, and investigated its structure, magnetic properties and magnetocaloric effect in detail.

The compound $\mathrm{GdCo}_{9} \mathrm{Si}_{2}$ was prepared by arc-melting a mixture of pure $\mathrm{Gd}$ (99.95 wt. \%), Co (99.99 wt. \%), and $\mathrm{Si}$ (99.99 wt. \%) in argon atmosphere. To ensure compositional homogeneity, the ingots were repeatedly melted at least four times. Before characterization, the ingots were wrapped in $\mathrm{Ta}$ foil and annealed at $1000{ }^{\circ} \mathrm{C}$ for 5 days. The structure of the samples was identified by Philips X'pert Pro MPD X diffractometer. The microstructure was observed using scanning electron microscope (SEM) with energy dispersive spectroscopy (EDS) attached. The temperature and magnetic field dependences of magnetization were measured by a physical properties measurement system (PPMS-9, Quantum Design Co.). The electrical resistance measurement was done by the four probe method using PPMS. The magnetic force microscopy (MFM) and atomic force microscopy (AFM) measurements were performed with a commercial scanning probe microscope (Asylum Research, Cypher).

The X-ray powder diffraction pattern indicates that $\mathrm{BaCd}_{11}$-type $\mathrm{GdCo}_{9} \mathrm{Si}_{2}$ (space group I41/amd) is the major phase, whilst there is still a minor phase of $\mathrm{Co}_{9} \mathrm{Si}$ (space group P63 $/ \mathrm{mmc}$ ). The refined unit cell parameters for $\mathrm{GdCo}_{9} \mathrm{Si}_{2}$ are: $a=7.932(2) \AA$ and $c=6.287(8) \AA$, respectively. The estimated content of $\mathrm{Co}_{9} \mathrm{Si}$ is about $7 \mathrm{wt}$. \%. The small amount of $\mathrm{Co}_{9} \mathrm{Si}$, with a Curie temperature higher than $900 \mathrm{~K}$, should make negligible contribution to the magnetic behavior of samples.

To confirm the contents of the impurity phase, the sample was also inspected using SEM/EDS. The back-scattered electrons image presented in Figure 1 confirms the composition of the major phase as 9.1 at. \% of Gd, 73.8 at. \% of Co, 


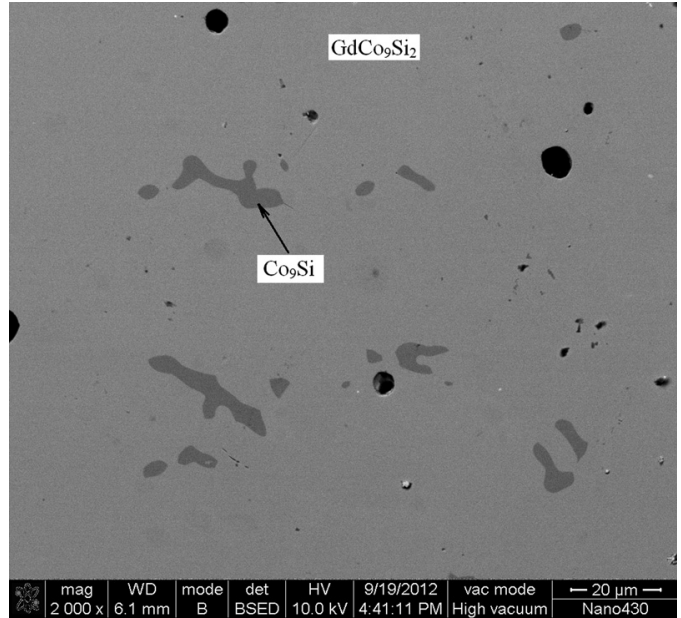

FIG. 1. Microstructure of the annealed sample. The major phase $\mathrm{GdCo}_{9} \mathrm{Si}_{2}$ and the impurities $\mathrm{Co}_{9} \mathrm{Si}$ are indicated. The black round areas correspond to the opened porosities.

and 17.1 at. $\%$ of $\mathrm{Si}$, close to the exact formula of $\mathrm{GdCo}_{9} \mathrm{Si}_{2}$. The stoichiometry of the gray zone is found to be $\mathrm{Co}_{9} \mathrm{Si}$. The black round areas correspond to the open porosities, which may be formed in arc melting processes. The fraction of $\mathrm{Co}_{9} \mathrm{Si}$ impurity was also estimated based on the SEM images to be less than $7 \%$, in good agreement with results from $\mathrm{X}$-ray diffraction.

Figure 2 shows the temperature dependence of zero field cooled (ZFC) and field cooled (FC) magnetizations for $\mathrm{GdCo}_{9} \mathrm{Si}_{2}$ compound. The transition temperatures are derived from the extreme values of $\mathrm{d} M / \mathrm{d} T$ vs $T$ curves shown as the inset of Fig. 2. It is clear that the compound exhibited magnetic ordering below $420 \mathrm{~K}\left(T_{\mathrm{SR} 2}\right)$, and a sharp drop of magnetic susceptibility was also evident at $93 \mathrm{~K}\left(T_{\mathrm{SR} 1}\right)$. A small thermal irreversibility between $\mathrm{ZFC}$ and $\mathrm{FC}$ curves is observed below $T_{\mathrm{SR} 2}$, which may be attributed to the domain-wall pinning effect. It is indicated that the compound undergoes two magnetic phase transitions with increasing temperature: an antiferromagnetic to ferrimagnetic near $T_{\mathrm{SR} 1}$ (Ref. 11) and then to paramagnetic transition at $T_{\mathrm{SR} 2}$. Above $T_{\mathrm{SR} 2}$, perfectly paramagnetic behavior was observed,

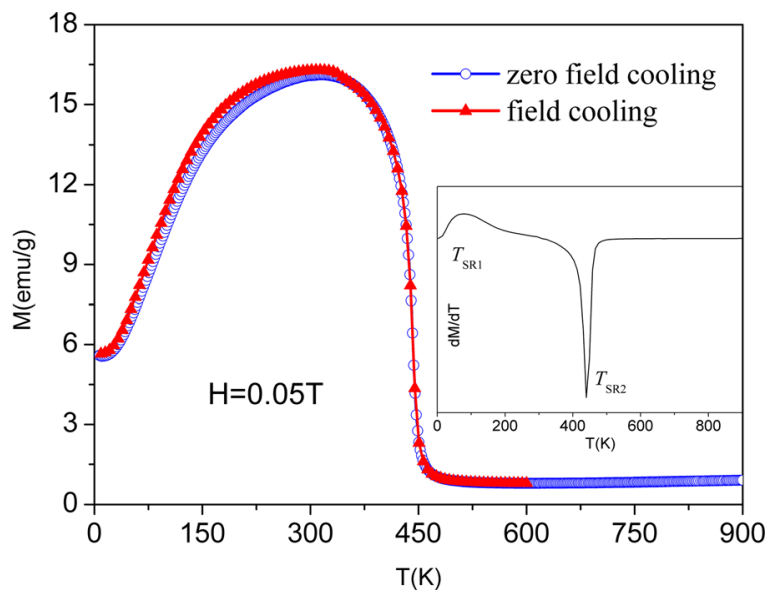

FIG. 2. Temperature dependences of $\mathrm{ZFC}$ and FC magnetization for $\mathrm{GdCo}_{9} \mathrm{Si}_{2}$ compound under a magnetic field of $0.05 \mathrm{~T}$. The inset is the differential of zero field cooling M-T curve. suggesting the negligible magnetic contribution of the $\mathrm{Co}_{9} \mathrm{Si}$ impurity even though its Curie temperature is higher than $900 \mathrm{~K}$. In compounds involving later rare-earth metals (REM) and transition metals (TM), the interaction between TM 3d electrons and REM 5d electrons dominates the magnetic behavior. Such interaction is generally negative and makes the overall $3 \mathrm{~d}-4 \mathrm{f}$ spin coupling antiferromagnetic. It has been reported that in the Co-rich Co-REM compounds the onset of long-range magnetic ordering is induced by the $3 \mathrm{~d}-3 \mathrm{~d}$ interaction in transition metals. ${ }^{10}$ Therefore, the abrupt increase of susceptibility when temperature is decreased below $420 \mathrm{~K}$ should result from the ordering of Co moments. Further decrease of temperature enhances the interaction of Gd lattice, but the antiferromagnetic interaction between Gd lattice and Co sublattice reduces the net moment. At low temperatures, both sublattices are saturated and the net moment is lowered to a minimum and invariable.

The saturation magnetization of $\mathrm{GdCo}_{9} \mathrm{Si}_{2}$ at $5 \mathrm{~K}$ is 9.4 emu/g, which equals $0.09 \mu_{\mathrm{B}}$ per formula unit, smaller than the value of a free $\mathrm{Gd}^{3+}$ ion $\left(7 \mu_{\mathrm{B}}\right)$, indicating antiferromagnetic coupling of the $\mathrm{Gd}$ and Co moments. With increasing temperature, the saturation magnetization gradually increases up to $29.5 \mathrm{emu} / \mathrm{g}$ at $300 \mathrm{~K}$, then decreases at higher temperatures. The insets of Figure 3 show the isothermal magnetization curves $M(H)$ for $\mathrm{GdCo}_{9} \mathrm{Si}_{2}$ with temperature from $34 \mathrm{~K}$ to $316 \mathrm{~K}$ and from $329 \mathrm{~K}$ to $585 \mathrm{~K}$. Based on the isothermal $M(H)$ curves and the Maxwell relation, the values of $-\Delta S_{\mathrm{M}}$ as a function of temperature for a maximum field of $5 \mathrm{~T}$ are calculated and shown in Figure 3. It is obvious that the positive and negative $-\Delta S_{\mathrm{M}}$ peaks for $\mathrm{GdCo}_{9} \mathrm{Si}_{2}$ compounds correspond to the first $\left(T_{\mathrm{SR} 1}\right)$ and second transitions $\left(T_{\mathrm{SR} 2}\right)$, respectively. The value of $-\Delta S_{\mathrm{Max}}$ at $T_{\mathrm{SR} 2}$ reaches 1.1 $\mathrm{J} \cdot \mathrm{kg}^{-1} \cdot \mathrm{K}^{-1}$ under $5 \mathrm{~T}$. It is comparable with those of some ferromagnetic materials, such as $\mathrm{Nd}_{0.9} \mathrm{Dy}_{0.1} \mathrm{Co}_{4} \mathrm{Al}^{12}$ and $\mathrm{Fe}_{75} \mathrm{Nb}_{10} \mathrm{~B}_{15}$ alloys. ${ }^{13}$ The other peak value of $-\Delta S_{\text {Max }}$ at $T_{\mathrm{SR} 1}$ is $-0.6 \mathrm{~J} \cdot \mathrm{kg}^{-1} \cdot \mathrm{K}^{-1}$ under $5 \mathrm{~T}$. The refrigerant capacity (RC) is obtained by integrating the area under the $-\Delta S_{\mathrm{M}}(\mathrm{T})$ curves, using the full temperature width at half maximum

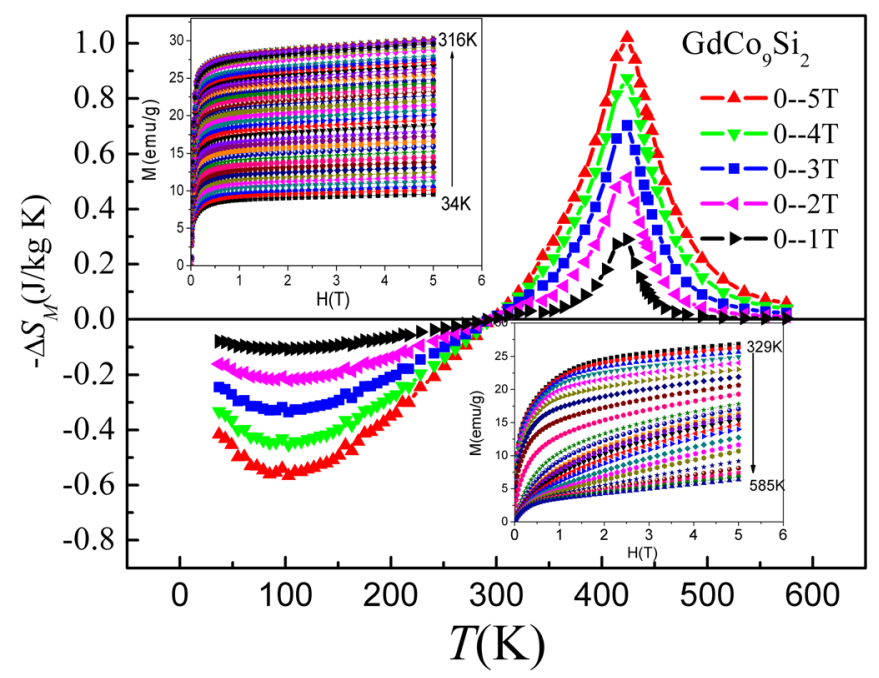

FIG. 3. Magnetic entropy change $-\Delta \mathrm{S}$ as a function of temperature in various applied magnetic fields change for $\mathrm{GdCo}_{9} \mathrm{Si}_{2}$ compound. The insets are isothermal magnetization curves. 

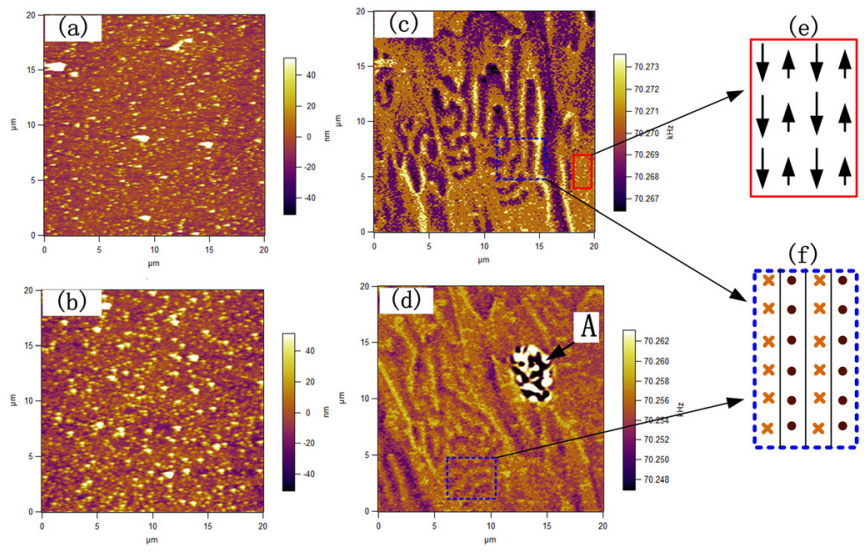

FIG. 4. The AFM (a), (b) and corresponding MFM (c), (d) morphology at room temperature, respectively. (e) and (f) The domain structure is sketched schematically as a top view and a cross-section corresponding to red solid and blue dashed box, respectively.

of the $-\Delta S_{\mathrm{M}}$ peak as the integration limits. The values of RC at $T_{\mathrm{SR} 2}$ are 22 and $62 \mathrm{~J} \cdot \mathrm{kg}^{-1}$ in a field of $2 \mathrm{~T}$ and $5 \mathrm{~T}$, respectively.

It is worth mentioning that temperature dependent electrical resistance $(R)$ measurements show a metalsemiconductor transition around $260 \mathrm{~K}$, as evidenced by a transition from an increasing $R$ with increasing temperature $(T)$, to a decreasing $R(T)$ curve, showing a peak at $260 \mathrm{~K}$. This peak is shifted to higher $T$ when magnetic field is applied, displacing the maximum to higher temperatures outside the experimental temperature range for $H=2 \mathrm{~T}$. A more detailed analysis of the possible influence of this transition on the magnetocaloric response is currently being undertaken and will be reported elsewhere.

From above discussion, one can justify that there is a ferrimagnetic phase near room temperature in $\mathrm{GdCo}_{9} \mathrm{Si}_{2}$ compound. In order to understand the magnetic domain structure, AFM and MFM experiments were performed at room temperature in the tapping or lift mode, as shown in Figure 4. Figure 4(a) presents the AFM image of the sample. The corresponding MFM image, shown in Figure 4(b), is characterized by the stripe and grid structures of $1 \mu \mathrm{m}$ wide, as shown in blue dashed box. With knowledge about the ferrimagnetic characterization and the presented AFM micrographs, the magnetic domains are sketched schematically as a top view and cross-section in Figure 4(f). The magnetic moments might display alternating in- and out-of-plane. Except for these structures, there are other type domain areas, such as red solid box, which could be ascribed to the alternating magnetic moments up- and down-of-plane, as shown in Figure 4(e). Figures 4(b) and 4(d) reveal the AFM and MFM images at other zones in the same sample, respectively. In addition to the stripe domain, there is a zone labeled "A" in which the magnetic domains become very strong. It could be associated to the ferromagnetic impurity phase $\mathrm{Co}_{9} \mathrm{Si}$.

The polycrystalline compound $\mathrm{GdCo}_{9} \mathrm{Si}_{2}$ with $\mathrm{BaCd}_{11}$ type structure (space group I41/amd) has been prepared by arc-melting. The structure, magnetic, and magnetocaloric properties of the compound have been investigated. Two magnetic transitions are observed: one from antiferromagnetic to ferrimagnetic $\left(T_{\mathrm{SR} 1}=93 \mathrm{~K}\right)$ and another to paramagnetic order $\left(T_{\mathrm{SR} 2}=420 \mathrm{~K}\right)$. There are two peak values in $-\Delta S_{M}(T)$, corresponding to $T_{\mathrm{SR} 1}$ and $T_{\mathrm{SR} 2}$, with magnitudes -0.6 and $1.1 \mathrm{~J} \cdot \mathrm{kg}^{-1} \cdot \mathrm{K}^{-1}$, respectively, for $\Delta H=5 \mathrm{~T}$. The magnetic domain structures show stripe and grid structures with about $1 \mu \mathrm{m}$ wide. It is interesting that the resistivity shows a metal-semiconductor transition at $\sim 260 \mathrm{~K}$ and to determine its origin theoretical calculations, X-ray photoelectron spectroscopy, and $\rho(T)$ studies under different magnetic field are in progress.

This work was financially supported by the Guangzhou Municipal Science and Technology Program (Grant No. 12F582080022), the Scientific Research Foundation for the Returned Overseas Chinese Scholars, State Education Ministry (Grant No. x2clB7120290), the Fundamental Research Funds for the Central Universities (Grant Nos. 2011ZM0014 and 2012ZZ0013), and the Guangdong Provincial Science and Technology Program (Grant Nos. 2010B050300008 and 2009B090300273).

${ }^{1}$ J. H. Belo, A. M. Pereira, J. Ventura et al., J. Alloys Compd. 529, 89-95 (2012).

${ }^{2}$ L. W. Li, K. Nishimura, H. Igawa et al., J. Alloys Compd. 509, 4198-4200 (2011).

${ }^{3}$ Z. G. Zheng, X. C. Zhong, H. Y. Yu et al., J. Appl. Phys. 109, 07A919 (2011).

${ }^{4}$ J. L. Zhang, Z. G. Zheng, W. H. Cao et al., J. Magn. Magn. Mater. 326, 157-161 (2013).

${ }^{5}$ X. C. Zhong, J. X. Min, Z. G. Zheng et al., J. Appl. Phys. 112(3), 33903 (2012).

${ }^{6}$ C. Mayer, E. Gaudin, S. Gorsse et al., J. Solid State Chem. 184(2), 325-330 (2011).

${ }^{7}$ J. Shen, J. F. Wu, and J. R. Sun, J. Appl. Phys. 106, 83902-83906 (2009).

${ }^{8}$ M. El-Hagary, H. Michor, and G. Hilscher, J. Magn. Magn. Mater. 322(19), 2840-2844 (2010).

${ }^{9}$ Y. L. Yaropolov, A. S. Andreenko, S. A. Nikitin et al., J. Alloys Compd. 509, S830-S834 (2011).

${ }^{10} \mathrm{M}$. El-Hagary, H. Michor, S. Özcan et al., J. Phys. Condens. Matter 18, 4567-4580 (2006).

${ }^{11}$ K. Madono, T. Matsui, S. Kosugi et al., Nucl. Instrum. Methods Phys. Res. B 267, 1604-1607 (2009).

${ }^{12}$ S. C. Ma, D. H. Wang, C. L. Zhang et al., J. Alloys Compd. 499, 7-10 (2010).

${ }^{13}$ J. J. Ipus, J. S. Blázquez, V. Franco et al., J. Appl. Phys. 105, 123922-123927 (2009). 